\title{
Radiosurgical treatment of recurrent esthesioneuroblastoma: case report
}

\begin{abstract}
Esthesioneuroblastoma (ENB) is a rare malignant neuroectodermal neoplasm originating from olfactory cells often with both of intracranial and extracranial growth. Since 1924 there are about 1200 reports of ENB worldwide. Treatment of ENB is still challenging caused by basal localization, infiltration of structures of anterior cranial fossa, very high local recurrence rate of $20-50 \%$ and possibility of distant metastases. In case of local recurrence is often applied a repeat resection or radiotherapy. However stereotactic radiosurgery is a non-invasive option. According to Gamma Knife Society database at the time of this report have been treated not more than 30 patients harboring ENB. In this case we report the use of Gamma Knife radiosurgery in management of recurrent ENB.
\end{abstract}

Volume 8 Issue | - 2018

Tokarev AS, Rak VA, Stepanov VN, Krylov VV

Sklifosovsky Research Institute of Emergency Care, Moscow, Russia

Correspondence: Rak VA, Sklifosovsky Research Institute of Emergency Care, Bolshaya Sukharevskaya sq. 3, Moscow, Russia, Tel 791532/2998, Email rakva@sklifos.ru

Received: November 07, 2017 | Published: February 21, 2018

Keywords: Esthesioneuroblastoma, Gamma knife, Radiosurgery

Abbreviations: ENB, Esthesioneuroblastoma; SRS, Stereotactic Radiosurgery; MRI, Magnetic Resonance Tomography; GTV, Gross Tumor Volume

\section{Introduction}

Esthesioneuroblastoma is an uncommon neuroectodermal tumor occurring with an incidence of 0.4 per 1 million and constitutes approximately $2-3 \%$ of sinonasal malignancies with no gender predilection. ${ }^{1,2}$ ENB was first described by Berger et al. ${ }^{3}$ in 1924 and since then approximately 1200 cases have been reported. ${ }^{3,4}$ The tumor arises from basal stem cells of sensory olfactory epithelium locating on superior nasal structures, cribriform plate and anterior skull base. ${ }^{4,5}$ The most common symptoms are nasal congestion and epistaxis, anosmia, rhinorrhea or lacrimation. ${ }^{6}$ Late clinical manifestation resulting from asymptomatic behavior of the tumor on early stages leads to high rate skull base involvement. Therefore the primary treatment modality is often a craniofacial resection, adjuvant external beam radiotherapy and chemotherapy. ${ }^{7}$ Despite combined treatment the risk of local recurrence is about 20-50\% ${ }^{4-7}$ Appliance of stereotactic radiosurgery (SRS) as a non-invasive treatment modality can be safe and effective in group of patients with local recurrence of ENB after surgery, especially in cases of deep located lesions. Here we present our experience of Gamma Knife SRS for an aggressive Kadish stage C ('spread beyond nasal and paranasal cavities') esthesioneuroblastoma involving right orbit, anterior cranial fossa and sphenopetroclival region.

\section{Case presentation}

Our patient, a 59-year-old man, presented 4 years ago with rhinitis. Examination of nasal cavity reviewed an exophytic mass in left superior meatus expanding to ethmoidal sinus. An incisional biopsy was performed by otolaryngologist. On histopathological examination, the tumor was composed of multiple primitive neuronal cells forming epithelioid structures with hyperchromatic nuclei and scanty cytoplasm. Neurofibrillary matrix was absent (Figure 1).

Serial MRI studies identified a solitary lesion with heterogeneous contrast enhancement in the nasal cavity, ethmoidal bulb and sphenoid sinus with destruction of cribriform plate and intracranial part of tumor. Due to large tumor volume and impossibility of total resection open surgery was not indicated. External beam radiotherapy was administered to skull base tumor (70Gy over 28 fractions). Additionally he received five rounds of doxorubicin and etoposide. Three years after this patient developed rapid vision loss in right eye. A local recurrence extending into right orbit, sella and sphenopetroclival region with destruction of clivus was identified on MRI. Tumor was resected through a craniofacial approach to prevent contralateral blindness. However significant tumor volume reduction was not achieved.

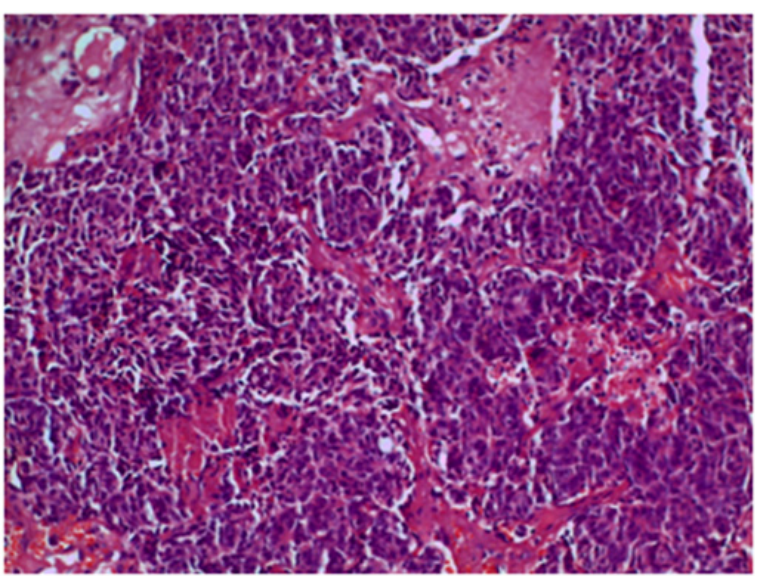

Figure I Hematoxylin and eosin stained slides (x200) showing olfactory neuroblastoma with primitive neural cells forming epithelioid structures and pseudoresettes without glandular differentiation, subtle fibrillary matrix.

One month later residual tumor was irradiated with Gamma Knife Perfexion (Elekta AB, Stockolm) (Figure 2a). The procedure started with application of the Leksell Model G stereotactic head frame (Elekta $\mathrm{AB}$ ) under local anesthesia. After frame placement, contrastenhanced high-resolution, thin-slice T1-weighted magnetic resonance imaging was performed for treatment planning. Gross tumor volume (GTV) was $57,26 \mathrm{~cm}^{3}$. ENB was treated administering a 18 -Gy margin dose at $50 \%$ isodose. Delivered dose was limited by GTV and proximity of left optical nerve, chiasm and brainstem to reduce the risk of radiation-induced complications. Overall 36 isocenters were used. At 6 month a significant tumor reduction was developed (Figure 2b). The patient had no signs of acute radiation toxicity or 
neurological deterioration. However, 9 months later new tumor foci were identified during follow-up MRI. Recurrent lesions were located near irradiated area (right orbit and nasal cavity) and distantly spreading on right frontal convexity duramater. Patient underwent a second Gamma Knife treatment (Figure 3). Total volume of 3 lesions was $14,25 \mathrm{~cm}^{3}$. The dose to margin was $14-18$ Gy at $50 \%$ isodose. Generalized seizures appeared three month later were successfully treated with anticonvulsants. Up to date there is no evidence of new recurrences or distant metastases.

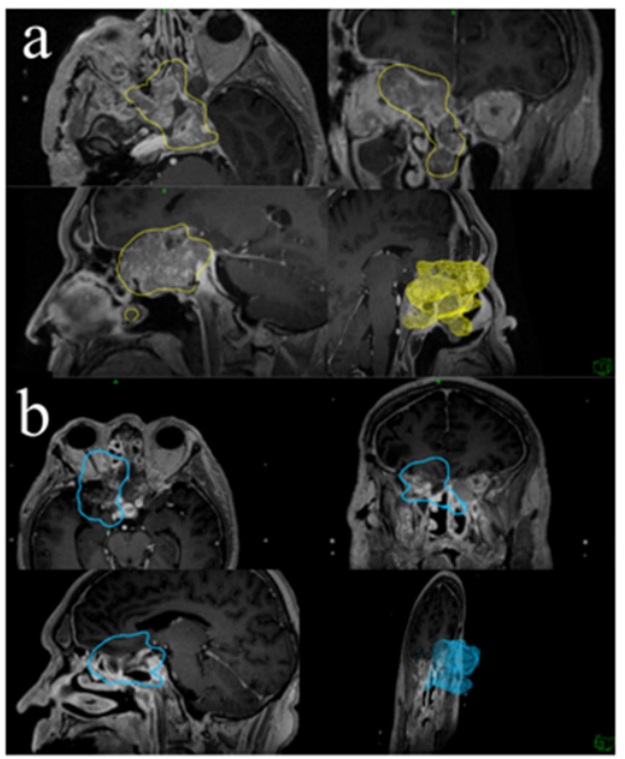

Figure 2 Gadolinium-enhanced TI-weighted magnetic resonance images in Leksell Gamma Plan 10.I demonstrating initial tumor volume at the time of SRS (a) and significant tumor reduction in 12 months (b). Yellow and blue lines show dose distribution.

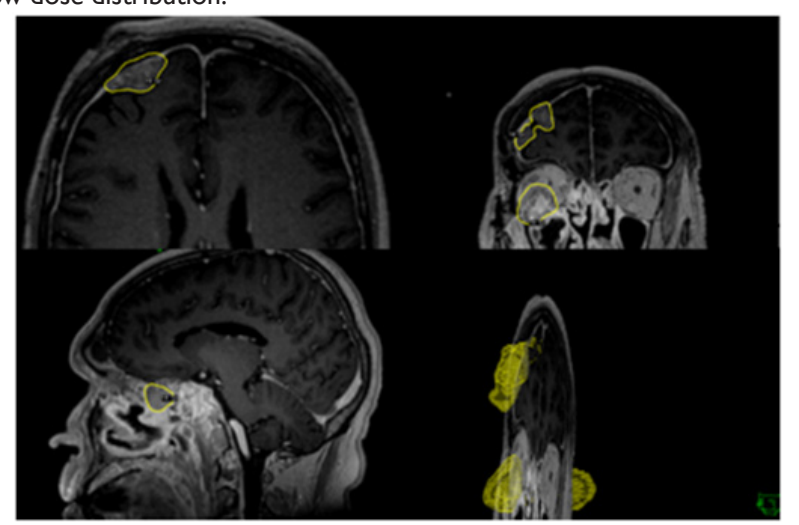

Figure 3 Gadolinium-enhanced TI-weighted magnetic resonance images in Leksell GammaPlan I0.I demonstrating multiple recurrent lesions in 16 months after first SRS. Yellow lines show dose distribution for second SRS.

\section{Discussion}

Most cases of EBN are diagnosed at later stages, at which appliance of surgery is often ineffective. For determination of possible treatment modalities and further prognosis the Kadish staging system can be used: stage A-disease limited to the nasal cavity, stage B-inclusion of the paranasal sinuses, stage $\mathrm{C}$-extension beyond the cribriform plate and paranasal sinuses and stage D (in modification of Morita et al..$^{9}$ ) the involvement of cervical lymph nodes and distant metastases. ${ }^{8,9} \mathrm{Five}$ year survival data for Kadish stages A, B, and C are $75 \%, 68 \%$ and $41 \%$ respectively. ${ }^{2}$ However, there is no common treatment strategy for Kadish $\mathrm{C}$ and $\mathrm{D}$ tumors. ${ }^{10}$ External beam radiation therapy (EBRT) and chemotherapy (etoposide and a platinum-based agent such as Cisplatin or Carboplatin) are usually administered in the adjuvant setting to patients with advanced disease. ${ }^{11,12}$ The Massachusetts General Hospital has an experience of craniofacial resection followed by proton beam irradiation. ${ }^{13}$

Only a few case series of radiosurgical treatment of these tumors are available in the literature. ${ }^{6,7,12,14}$ Van Gompel et al. ${ }^{7}$ reported a multiinstitutional experience of 4 radiosurgical centers including only 13 patients harboring EBM overall. Median dose to the tumor margin was 15 Gy with $92 \%$ rate of local control. Unger et al. ${ }^{6}$ reported a series of 14 patients received combined treatment: endoscopic surgery for the nasal and paranasal component and SRS (Marginal irradiation doses 15-34 Gy) for the intracranial component with excellent local control rate.

In this report we confirm high efficacy of SRS of recurrent EBM involving multiple intracranial structures and orbit. Gamma Knife radiosurgery can be applied to avoid surgical complications as CSF leak, meningitis, pneumocephalus, abscess formation or osteomyelitis. High dose gradient between tumor and healthy tissues and high beam conformity decrease risk of radiation toxicity after EBRT and in case of involvement of deep structures such as cavernous sinus or clivus. SRS is also highly recommended when identifying contraindications to open surgery. However, despite new developed treatment regimens including radiosurgery, the overall long-term survival rate of patients with ENB recurrence remains poor.

\section{Acknowledgements}

None.

\section{Conflicts of interest}

All authors certify that they have no affiliations with or involvement in any organization or entity with any financial interest (such as honoraria; educational grants; participation in speakers' bureaus; membership, employment, consultancies, stock ownership, or other equity interest; and expert testimony or patent-licensing arrangements), or non-financial interest (such as personal or professional relationships, affiliations, knowledge or beliefs) in the subject matter or materials discussed in this manuscript.

\section{References}

1. Theilgaard SA, Buchwald C, Ingeholm P, et al. Esthesioneuroblastoma: a Danish demographic study of 40 patients registered between 1978 and 2000. Acta Otolaryngol. 2003;123(3):433-439.

2. Wenig BM. Atlas of Head and Neck Pathology. ( ${ }^{\text {rd }}$ edn), Elsevier, Philadelphia, USA. 2016;pp.148-155.

3. Berger L, Luc G, Richard D. L'esthésioneuroépithéliome olfactif. Bull Assoc Fr Etud Cancer. 1924;13:410-421.

4. Gandhoke CS, Dewan A, Gupta D, et al. A rare case report of mixed olfactory neuroblastoma: Carcinoma with review of literature. Surg Neurol Int. 2017;8:83.

5. Gore MR, Zanation AM. Salvage Treatment of Local Recurrence in Esthesioneuroblastoma: A Meta-analysis. Skull Base. 2011;21(1):1-6.

6. Unger F, Haselsberger $\mathrm{K}$, Walch $\mathrm{C}$, et al. Combined endoscopic surgery and radiosurgery as treatment modality for olfactory neuroblastoma (esthesioneuroblastoma). Acta Neurochir (Wien). 2005;147(6):595-601.

7. Van Gompel JJ, Carlson ML, Pollock BE, et al. Stereotactic radiosurgical salvage treatment for locally recurrentesthesioneuroblastoma. Neurosurgery. 2013;72(3):332-339. 
8. Kadish S, Goodman M, Wang CC. Olfactory neuroblastoma. A clinical analysis of 17 cases. Cancer. 1976;37(3):1571-1576.

9. Morita A, Ebersold MJ, Olsen KD, et al. Esthesioneuroblastoma: Prognosis and management. Neurosurgery. 1993;32(5):706-714.

10. Palejwala SK, Sharma S, Le CH, et al. Complications of Advanced Kadish Stage Esthesioneuroblastoma: Single Institution Experience and Literature Review. Cureus. 2017;9(5):e1245.

11. Loy AH, Reibel JF, Read PW, et al. Esthesioneuroblastoma: continued follow-up of a single institution's experience. Arch Otolaryngol Head Neck Surg. 2006;132(2):134-138.
12. McLean JN, Nunley SR, Klass C, et al. Combined modality therapy of esthesioneuroblastoma. Otolaryngol Head Neck Surg. 2007;136(6):998-1002.

13. Herr MW, Sethi RK, Meier JC, et al. Esthesioneuroblastoma: an update on the massachusetts eye and ear infirmary and massachusetts general hospital experience with craniofacial resection, proton beam radiation, and chemotherapy. J Neurol Surg B Skull Base. 2014;75(1):58-64.

14. Dinca EB, Radatz MW, Rowe J, et al. Gamma Knife ${ }^{\circledR}$ radiosurgery for recurrent intracranial olfactory neuroblastoma (esthesioneuroblastoma): a case report. J Med Case Rep. 2012;6:240. 